\title{
Short Term Prediction of Electric Vehicle Charging Load Based on Optimized Genetic Algorithm
}

\author{
Qu TianYi1, a \\ ${ }^{1}$ School of Management, XuZhou University of Technology, Xuzhou, Jiangsu, China 221008 \\ ajdbh2001@163.com
}

Keyword: Electric vehicle; Load forecasting; Genetic algorithm; BP neural network

\begin{abstract}
With the continuous attention and promotion of electric vehicles, governments of electric vehicles have made great progress. However, due to the randomness and unpredictable nature of electric vehicle charging, it will have a certain impact on the power system. To effectively predict the charging load of electric vehicles can effectively alleviate the impact of electric vehicle charging on the distribution network to a certain extent. This paper proposes a method to predict the charging load of electric vehicles by using the genetic algorithm to optimize the numerical value and weight threshold of the number of the hidden layer units of the neural network structure, and compares it with the BP neural network prediction method. The experimental data show that the prediction method has higher prediction accuracy.
\end{abstract}

\section{Introduction}

As a product of the times, the rechargeable car can not only alleviate the shortage of resources, but also reduce the emission of greenhouse gas to a large extent, which is beneficial to environmental protection $[1,2,3]$. However, the wide access of electric vehicle charging load will have a great impact on the operation of the power grid. A large number of electric vehicles connected to the power grid will have a great impact on the distribution network structure and related equipment. At the same time, it may trigger a new round of load growth, and a large number of charging facilities will put forward more requirements for upgrading and upgrading of the power grid. Due to the strong randomness of the behavior of electric vehicle users and the time and space of charging, the charging load of the electric vehicle has great uncertainty, which makes the control difficulty of the power grid greatly increase. Electric vehicle charging facilities contain a large number of nonlinear components affecting the power quality of the power grid and other $[4,5,6]$. Therefore, the research on the access of electric vehicle charging facilities will play a positive role in promoting the development of electric vehicles.

On the basis of considering the historical curve of electric vehicle charging load and the main factors affecting the charging load, this paper proposes a method to predict the charging load of electric vehicles by using the genetic algorithm to optimize the number of the hidden layer units of the neural network structure and the weight threshold. The empirical evidence has ahigher prediction accuracy.

\section{An optimization algorithm based on combination of genetic algorithm and neural network}

The training process of connection weights of neural networks is a process of decreasing errors. The connection weights of neural networks are generally random initialized into $[-0.5,0.5]$ interval random numbers. This random number has great influence on network training. Gradient descent method is widely used in neural networks. It is very sensitive to the initial value of connection weights. Because different initial values will produce different training effects, there will be different error values, such as the improper initial value will cause the network concussion so that the network cannot converge, and may make the network converge slow so that the training time is very long, and the same pattern may also make the network fall into the local minimum. A value that cannot reach the global optimal value. In spite of this, there is no mature theory to effectively 
explain the network connection weights, and a relatively good connection right can be obtained by optimizing the genetic algorithm.

It is very important to determine the number of hidden layer elements in neural network, because this is the most critical factor affecting the quality of the network, and there is no mature theoretical support. At present, the empirical formula method and enumeration method [8] will affect the prediction accuracy and increase the prediction time.

Through the understanding and analysis of the single optimization process, the genetic algorithm is used to optimize the network structure and the connection right. The implementation steps of the algorithm are as follows Fig.1:

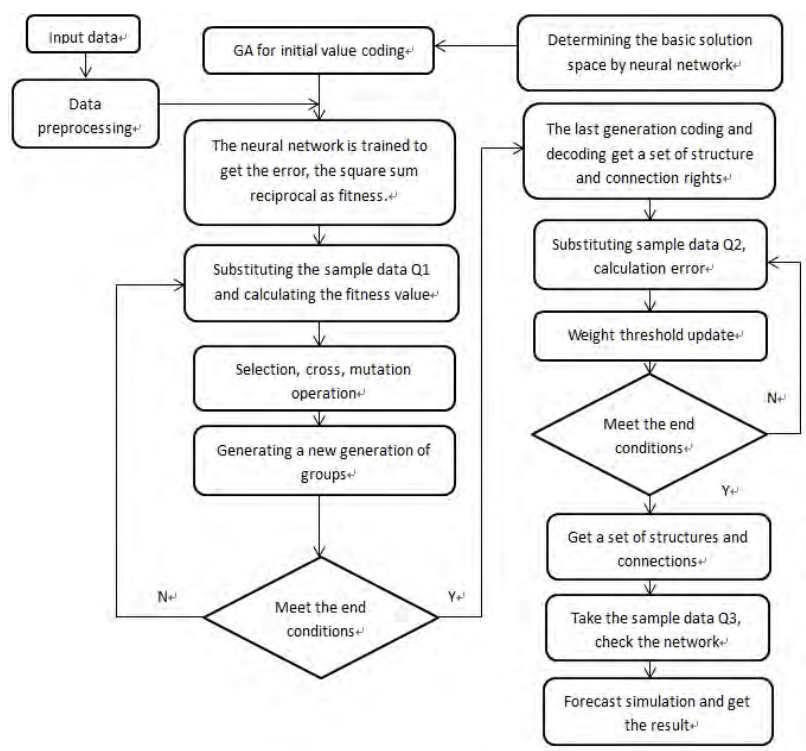

Figure1. Detailed optimization flow figure

\section{Implementation Method Based on Combinatorial Optimization Algorithm}

After a large number of data analysis and comprehensive consideration of the main influence factor [11] of electric vehicle charging load, the number of input layers of the neural network is 4 , which are the historical load of electric vehicle charging, the corresponding historical temperature, the corresponding history weather and the travel law, and the number of output layers is 1 , which is the charging load of one day that needs to be predicted.

Taking the data of the charging load of a bus charging station in 2017, and combining the data of temperature, weather and holiday in the year of the year, the load forecast of the charging station is analyzed in accordance with the above steps.

In 2017, three days (Day1, Day2) were selected randomly to verify the prediction method proposed in this paper. The following is a comparison between daily load forecasting curve and daily load actual curve.

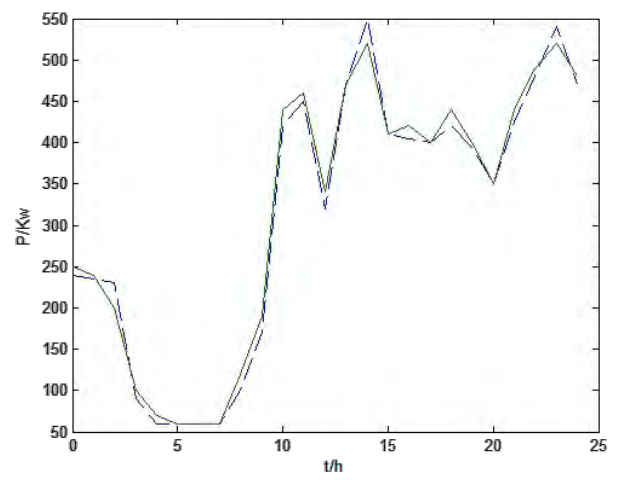

Figure 2. Day1 comparison between the actual value and the predicted value 


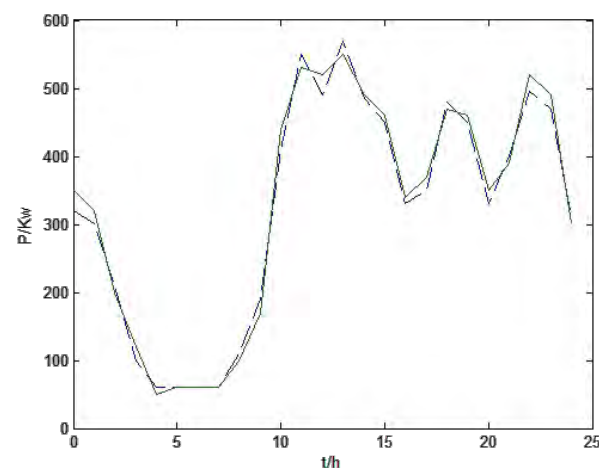

Figure 3. Day2 comparison between the actual value and the predicted value

\section{Conclusion}

In this paper, the genetic algorithm and neural network are combined to make up the defects of the local minimum value of the neural network, and give full play to the advantages of the two, so that the electric vehicle charging daily load forecasting is more accurate. As can be seen from figures 2 , 3 , and 4 , the charging time of electric vehicles is more concentrated in two periods, and with the increasing of the number of electric vehicles, the charging load will increase continuously. After the charge load is superimposed on the conventional load of the power grid, it will bring a series of effects, such as increasing peak valley difference, and harmonics. A large influx of and so on. The short-term load forecasting of electric vehicle based on neural network optimization is helpful to the short-term load dispatching of the power grid, but it can not provide substantial reference to the long-term planning of the power grid.

\section{Reference}

[1] Yang Jingwei. Prediction method of electric vehicle charging load and charging control strategy.[Master's Thesis].Hunan University, 2015.

[2] Li Yuzhe. Multi factor prediction model of electric vehicle load and its impact on power grid [Master's Thesis].Chongqing University, 2016.

[3] Li Haijuan. Short term load forecasting for smart distribution network of electric vehicles [Master's Thesis].Southeast University, 2015.

[4] Li Zhihui. Influence of electric vehicle charging station on power grid [Master's Degree Thesis]. Shandong University, 2013.

[5] Wang Jian, Wu Kuihua, Liu Zhizhen, Wu Kuizhong, Sun Wei. Influence of electric vehicle charging on power distribution network load and order control research [J]. power automation equipment, 2013,08:47-52.

[6] Bass R, Harley R, Lambert F, et al. Residential harmonic loads and EV charging [C]. Power Engineering Society Winter Meeting, Columbus USA, 2001, pp.803-808.

[7] Zhu Haitao. Thoughts on the prediction of the charging load of electric vehicles considering time and space distribution [journal articles] - technology and innovation

[8] Liu Qing, Qi Zhong. Electric vehicle load forecasting model based on Montcarol [J]. electric power science and engineering, 2014,30 (10): 14-19.

[9] Yang Shaobing, Wu Shen Li, Jiang Jiuchun, et al. Load modeling method for electric vehicle charging station [J]. power grid technology, 2013,37 (5): 1190-1195.

[10] Jian Sheng. Genetic algorithm based BP neural network weather prediction modeling [Master's Thesis]. Guangxi Normal University, 2001. 